\title{
Overexpression of G3BP1 facilitates the progression of colon cancer by activating $\beta$-catenin signaling
}

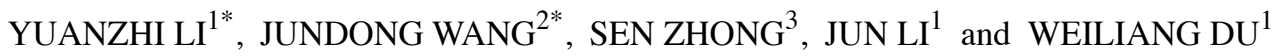 \\ ${ }^{1}$ Traditional Chinese Medicine Department, The Affiliated Hospital of Southwest Medical University, \\ Luzhou, Sichuan 646000; ${ }^{2}$ Gastroenterology Department and ${ }^{3}$ Infectious Department, \\ Hospital of Chengdu University of Traditional Chinese Medicine, Chengdu, Sichuan 610000, P.R. China
}

Received September 5, 2019; Accepted August 28, 2020

DOI: $10.3892 / \mathrm{mmr} .2020 .11527$

\begin{abstract}
Ras-GTPase-activating protein SH3 domain-binding protein 1 (G3BP1) has been reported to be of importance in the occurrence and development of colon cancer. However, the underlying mechanisms remain largely unknown. Therefore, the aim of the present study was to investigate the role of Wnt/ $\beta$-catenin signaling in G3BP1-mediated colon cancer progression. The expression of G3BP1 in colon tissues and cells was detected via reverse transcription-quantitative PCR, western blotting and immunohistochemistry. Gain-of-function assays were performed in colon cancer RKO cells, which have a relatively low expression of G3BP1, while loss-of-function assays were performed in SW620 colon cancer cells, which have a relatively high expression of G3BP1. Cell proliferation, apoptosis and tumorigenesis were assessed using Cell Counting Kit-8, flow cytometry and tumor-bearing mice assays, respectively. The results demonstrated that G3BP1 expression was significantly upregulated in colon cancer tissues and cells compared with healthy colon tissues and cells. It was found that high expression of G3BP1 was closely associated with the poor prognosis and advanced clinical process in patients with colon cancer. Overexpression of G3BP1 in RKO cells enhanced their proliferative ability and decreased their apoptosis tendency, while knockdown of G3BP1 inhibited SW620 cell proliferation and induced apoptosis. In addition, G3BP1 interacted with $\beta$-catenin and upregulated its expression and nuclear accumulation. It was identified that $\beta$-catenin knockdown abolished the effects of G3BP1 on the enhancement of cell proliferation in vitro and tumor formation in vivo, as well
\end{abstract}

Correspondence to: Dr Yuanzhi Li, Traditional Chinese Medicine Department, The Affiliated Hospital of Southwest Medical University, 25 Taiping Street, Luzhou, Sichuan 646000, P.R. China E-mail: yuanzhili5945@163.com

*Contributed equally

Key words: Ras-GTPase-activating protein SH3 domain-binding protein 1 , proliferation, apoptosis, tumorigenesis, $\beta$-catenin, colon cancer as the inhibition of cell apoptosis. In conclusion, the present study demonstrated that G3BP1 promoted the progression of colon cancer by activating $\beta$-catenin signaling, which provided novel evidence for the role of G3BP1 in colon cancer.

\section{Introduction}

Colon cancer, which occurs in digestive system, is one of the most common types of malignant cancer and is the second cause of cancer-associated mortality worldwide, causing $\sim 600,000$ cases of mortality per year (1). It is reported that $>1.4$ million people are diagnosed with this disease in the USA, and an additional 134,490 cases are diagnosed annually in the USA (2). Although great progress has been achieved in multimodality therapy, patients with colon cancer at an advanced stage still have poor outcomes. For instance, it has been reported that $>90 \%$ of patients with colon cancer at stage I can survival for $\geq 5$ years, but this is declined to $\sim 10 \%$ in patients with stage IV colon cancer (3). Dysregulated genes serve a crucial role in the progression of colon cancer, but there are numerous unidentified genes or known genes whose functions are yet to be elucidated in colon cancer (4).

Ras-GTPase-activating protein SH3 domain-binding proteins (G3BPs) are a class of RNA-binding proteins and contain two homologous proteins (G3BP1 and G3BP2) (5). All G3BPs include a RNA recognition motif, and have been identified to exert both mRNA-stabilizing and mRNA-degrading roles $(6,7)$. Although both G3BP1 and G3BP2 are reported to be involved in carcinogenesis (8), G3BP1 appears to have been studied more extensively. For example, Zheng et al (9) revealed that elevated expression of G3BP1 predicted poor prognosis in patients with non-small cell lung cancer after surgical resection. Furthermore, Winslow et al (10) compared the effects of G3BP1 and G3BP2 on breast cancer progression, and the results indicated that G3BP1 to a larger extent than G3BP2 influenced mRNA expression levels of peripheral myelin protein 22 (PMP22), a gene regulated by G3BP1 and that potentially mediates G3BP1 effects on cell proliferation enhancement. G3BP1 has been demonstrated to be involved in multiple biological processes, including RNA metabolism (11), cell proliferation (12), apoptosis (13), motility and invasion (7). Moreover, G3BP1 upregulation has been frequently observed in several type of cancer, such as breast cancer (14), 
gastric cancer (15), lung cancer (16) and colon cancer (17). Zhang et al (18) reported that knockdown of G3BPs (G3BP1 and G3BP2) significantly inhibited the proliferation of colon cancer HCT116 cells and improved sensitivity to cisplatin, as well as weakened cell tumorigenesis, suggesting that G3BP1 serves a crucial role in the progression of colon cancer. However, the mechanisms via which G3BP1 facilitates colon cancer progression and the clinical values of $\mathrm{G} 3 \mathrm{BP} 1$ require further investigation.

Previous studies have revealed that aberration of the Wnt/ $\beta$-catenin signaling pathway significantly contributes to the malignant transformation of colon cancer $(19,20)$. Inhibition of $\mathrm{Wnt} / \beta$-catenin is suggested to be an anti-tumor mechanism in colon cancer (21). In the absence of Wnt, the phosphoprotein scaffold Dishevelled3 (Dsh3) promotes the destabilization of $\beta$-catenin 1 mRNA (22). The $\beta$-catenin protein is controlled by a protein complex that consists of axis inhibitor (Axin), adenomatous polyposis coil (APC), casein kinase $1 \alpha$ and glycogen synthase kinase $3 \beta$, and is confined in the cytoplasm (22). However, $\beta$-catenin is increased in cytoplasm and eventually translocates into the nucleus once Wnt signaling is activated, inducing the transcription of target genes, including c-myc, a cell proliferation-associated gene (21). G3BP1 has been identified to be a Dsh-associated protein that is methylated in response to Wnt3a and negatively regulates $\beta$-catenin expression (23). However, the role of Wnt/ $\beta$-catenin signaling in G3BP1-mediated colon cancer progression remains unknown.

The aim of the present study was to investigate whether G3BP1 promoted the progression of colon cancer by regulating the Wnt/ $\beta$-catenin signaling pathway, which could further reveal the role of G3BP1 in colon cancer and help to identify novel targets for colon cancer treatment.

\section{Materials and methods}

Colon tissue specimens. All primary colon cancer tissues and matched healthy colon tissues $(\geq 3.5 \mathrm{~cm}$ from the cancer tissue) were obtained from 92 patients (age range, 38-73 years; 50 male and 42 female patients) with colon cancer who received a colectomy between January 2010 and January 2017 in The Affiliated Hospital of Southwest Medical University. All patients signed the informed consent forms and received colectomy as the first treatment method. The fresh tissues were immediately immersed in liquid nitrogen and stored at $-80^{\circ} \mathrm{C}$ until further analysis.

Experiments involving human samples were performed in accordance with the Declaration of Helsinki and were approved by The Ethical Committee of The Affiliated Hospital of Southwest Medical University.

Immunohistochemistry (IHC). For IHC, routine three-step procedures were performed as previously described (24) with a primary antibody against G3BP1 (cat. no. ab56574; Abcam). Tissue samples were fixed in $10 \%$ neutral formalin for 2 days at room temperature and embedded in paraffin, after which 4- $\mu \mathrm{m}$ sections were cut and mounted onto slides. Slides were incubated at $56^{\circ} \mathrm{C}$, deparaffinized in xylene and dehydrated in a graded series of alcohol. Heat-induced antigen retrieval was carried out with sodium citrate ( $\mathrm{pH}$ 6.7) in a pressure-cooker for
30 min. Following washing with PBS, the slides were blocked with $\mathrm{PBS}+5 \%$ goat serum for $1 \mathrm{~h}$ at room temperature, then and incubated overnight at $4^{\circ} \mathrm{C}$ with primary antibody against G3BP1 at a dilution of 1:150. Next, a HRP-conjugated secondary antibody (cat. no. ab5879; Abcam) was added for $30 \mathrm{~min}$ at room temperature. The staining was examined under a light microscope (Olympus Corporation; magnification, $\mathrm{x} 100$ ).

The expression of G3BP1 was determined by three pathologists in a blinded manner by multiplying the staining extent and intensity scores. The extent of positively stained cells was scored as: i) 0 for $0-5 \%$; ii) 1 for $6-25 \%$; iii) 2 for $26-50 \%$; iv) 3 for $51-75 \%$; and v) 4 for $76-100 \%$. The staining intensity was scored as: i) 0 for negative staining; ii) 1 for weak staining; iii) 2 for moderate staining; iv) and 3 for strong staining. A total score of lower or equal to the average score calculated from all samples was defined as low G3BP1expression, and high expression otherwise.

Cell culture. A normal human intestinal epithelial cell (HIEC) line, and the colon cancer cell lines SW620, LoVo, RKO, COLO 205 and HCT116 were obtained from the Cell Bank of the Chinese Academy of Sciences. HIECs were cultured in medium containing 90\% DMEM-H (Thermo Fisher Scientific, Inc.) and 10\% FBS (HyClone; Cytiva). LoVo cells were cultured in F-12K medium (Thermo Fisher Scientific, Inc.) supplemented with $10 \%$ FBS. RKO cells were cultured in $90 \%$ Eagle's Minimum Essential Medium (Thermo Fisher Scientific) and 10\% FBS. SW620, HCT116 and COLO 205 cells were cultured in RPMI-1640 (Thermo Fisher Scientific, Inc.) medium containing $10 \% \mathrm{FBS}$. All cells were maintained in a $5 \% \mathrm{CO}_{2}$ atmosphere at $37^{\circ} \mathrm{C}$.

Alteration of gene expression. To overexpress G3BP1, colon cancer cells were infected with the lentivirus vector (Vector-G3BP1; Shanghai GenePharma Co., Ltd.) using $5 \mu \mathrm{g} / \mathrm{ml}$ polybrene (Hanbio Biotechnology Co., Ltd.) with an MOI of 10, and the infected cells were incubated with G401 $(100 \mu \mathrm{g} / \mathrm{ml})$ for 14 days at $37^{\circ} \mathrm{C}$ to establish the stably infected cells.

To silence $\beta$-catenin expression, colon cancer cells were infected with the lentivirus vector [short hairpin RNA (sh)- $\beta$-catenin; OriGene Technologies, Inc.] and puromycin $(100 \mu \mathrm{g} / \mathrm{ml})$ was applied to select the stably infected cells at $37^{\circ} \mathrm{C}$ for 14 days.

To knockdown G3BP1 expression, colon cancer cells were transfected with the small interfering (si)RNAs targeting G3BP1 (si-G3BP1; OriGene Technologies, Inc.) using Lipofectamine ${ }^{\circledR} 2000$ reagent (Thermo Fisher Scientific, Inc.) according to the manufacturer's instructions. The sequences were as follows: i) si-G3BP1-1, 5'-CCACACCAAGATTCG CCAT-3'; ii) si-G3BP1-2,5'-GGAGATTCATGCAAACGTT-3'; iii) si-G3BP1-3: 5'-GGAGGAGTCTGAAGAAGAA-3'; and iv) si-NC: 5'-CCAAACCTTAGCGCACCAT-3'. Vector-NC, sh-NC and si-NC (OriGene Technologies, Inc.) were used as the negative controls for Vector-G3BP1, sh- $\beta$-catenin and si-G3BP1, respectively. After 48 h of transfection/infection, the cells were collected for subsequent experiments.

Reverse transcription-quantitative PCR (RT-qPCR). Total RNA was extracted from snap-frozen tissues or cultured cells using TRIzol ${ }^{\circledR}$ reagent (Takara Biotechnology Co., Ltd.) 
based on the manufacturer's instructions. The obtained RNA samples were quantitated using a NanoDrop 2000 system (NanoDrop Technologies; Thermo Fisher Scientific, Inc.) and identified using $2 \%$ agarose gel electrophoresis. Next, the first-strand cDNA was synthesized with random primers (Beijing Solarbio Science \& Technology Co., Ltd.) using the High-Capacity cDNA Reverse Transcription Kit (Thermo Fisher Scientific, Inc.), and RT-qPCR was performed with a SuperScript ${ }^{\circledR}$ III One-Step RT-PCR system with Platinum Taq Mix (Thermo Fisher Scientific, Inc.), according to the manufacturer's instructions. Reaction conditions were as follows: i) $94^{\circ} \mathrm{C}$ for $5 \mathrm{~min}$; ii) 40 amplification cycles at $94^{\circ} \mathrm{C}$ for $30 \mathrm{sec}$, $57^{\circ} \mathrm{C}$ for $30 \mathrm{sec}$ and $72^{\circ} \mathrm{C}$ for $30 \mathrm{sec}$; and iii) $72^{\circ} \mathrm{C}$ for $5 \mathrm{~min}$. The mRNA expression of GAPDH was used as the endogenous control. The mRNA levels were calculated by using the $2^{-\Delta \Delta \mathrm{Cq}}$ method (25). The following primers were used: G3BP1 forward, 5'-CAGCCGCGTAGGTTGAATTG-3' and reverse, 5'-AGAGAGAAGCCCCATCACCT-3'; GAPDH forward, 5'-CCACTAGGCGCTCACTGTTCTC-3' and reverse, 5'-ACT CCGACCTTCACCTTCCC-3'.

Western blotting. Total proteins extracted from cells and colon tissues were obtained using RIPA lysis buffer (Beijing Solarbio Science \& Technology Co., Ltd.) according to the manufacturer's instructions. The nuclear and cytoplasmic protein samples were isolated from cells using a Nuclear and Cytoplasmic Protein Extraction kit (Beyotime Institute of Biotechnology) following the manufacturer's instructions. A total of $30 \mu \mathrm{g}$ proteins from different groups were subjected to $10 \%$ SDS-PAGE and separated at $85 \mathrm{~V}$ for $30 \mathrm{~min}$ and $120 \mathrm{~V}$ for 90 min, which were then transferred onto PVDF membranes (Thermo Fisher Scientific, Inc.). After being blocked with 5\% non-fat milk for $1 \mathrm{~h}$ at room temperature, the membranes were incubated with the primary antibodies, including anti-G3BP1 (cat. no. ab56574; Abcam), Axin (cat. no. ab32197; Abcam), $\beta$-catenin (cat. no. MA1-300; Thermo Fisher Scientific, Inc.), Frizzled (Frz; cat. no. AF1617; R\&D Systems, Inc.), Dsh (cat. no. TA300981; OriGene Technologies, Inc.), TCF/LEF transcription factor family (TCF; cat. no. 9383; Cell Signaling Technology, Inc.), c-myc (cat. no. M4439; Sigma-Aldrich; Merck KGaA), APC (cat. no. ab15270; Abcam), Lamin B1 (cat. no. ab16048; Abcam) and GAPDH (cat. no. 60004-1-Ig; Wuhan Sanying Biotechnology) overnight at $4^{\circ} \mathrm{C}$. The following day, the membranes were washed four times ( 8 min each time) with TBS-Tween-20 [1\% (v/v)] and incubated with horseradish peroxidase (HRP)-conjugated secondary antibodies (1:10,000 dilution; cat. nos. SA00001-1 and SA00001-2; Wuhan Sanying Biotechnology) at room temperature for $1 \mathrm{~h}$. The protein bands were visualized with using a chemiluminescent HRP substrate (EMD Millipore). ImageJ software (version 1.48; National Institutes of Health) was used to assess the relative expression levels of proteins.

Co-immunoprecipitation (Co-IP). The interaction between G3BP1 and $\beta$-catenin proteins was assessed using a Co-IP assay. SW620 and RKO cells infected with Vector-G3BP1 or Vector-negative control (NC) were rinsed with cold PBS and lysed in IP lysis buffer, followed by centrifugation at $10,000 \mathrm{x} \mathrm{g}$ at $4^{\circ} \mathrm{C}$ for $30 \mathrm{~min}$. Then, $200 \mu \mathrm{g}$ proteins from each sample were incubated with Dynabeads ${ }^{\circledR}$ protein G (Thermo Fisher
Scientific, Lnc.) for $1 \mathrm{~h}$ at room temprature, and incubated with $2 \mu \mathrm{g}$ of anti-G3BP1 (cat. no. ab181150; Abcam) or anti- $\beta$-catenin (cat. no. ab16051; Abcam) antibody overnight at $4^{\circ} \mathrm{C}$. Anti-IgG antibody (cat. no. ab182931) was used as a negative control. This was followed by incubation with Dynabeads ${ }^{\circledR}$ protein $G$ for another $1 \mathrm{~h}$ at room temperature. Subsequently, the immunocomplex was washed five times with IP lysis buffer (Thermo Fisher Scientific, Inc.) and then subjected to western blot analysis with anti- $\beta$-catenin (cat. no. MA1-300; Thermo Fisher Scientific, Inc.) or anti-G3BP1 (cat. no. ab56574; Abcam) antibodies at 1:2,000 dilution.

Cell proliferation and apoptosis detection. SW620 and RKO cells in the logarithmic phase with lentivirus infection, siRNA transfection or without were seeded into 96-well plates at 3,000 cells/well density and cultured at $37^{\circ} \mathrm{C}$. Cell proliferation was tested using the Cell Counting Kit-8 (CCK-8; Dojindo Molecular Technologies, Inc.) and the absorbance at the wavelength of $450 \mathrm{~nm}$ was measured according to the manufacturer's instructions after 1, 2, 3, 4 or 5 days of cell inoculation.

Apoptotic rates (the percentage of early- and late-apoptotic cells) were measured in SW620 and RKO cells with lentivirus infection, siRNA transfection or without using the Annexin V-FITC Apoptosis Detection kit I (BD Biosciences) and assessed by flow cytometry. The cells were suspended in $1 \mathrm{X}$ binding buffer and incubated with $5 \mu \mathrm{l}$ Annexin V-FITC and $5 \mu \mathrm{l} \mathrm{PI}$ in the dark for $10 \mathrm{~min}$. Cell apoptotic rates were detected on a BD FACSCanto II instrument (BD Biosciences) and analyzed using FlowJo 7.6 software (FlowJo LLC).

Mouse xenografts of human colon cancer cells. Mouse xenografts assays were used to evaluate the effect of G3BP1 and $\beta$-catenin on cell tumorigenesis, and were performed as previously reported (26). A total of 30 male nude mice (weight, 12-14 g; age, 4-6 weeks) were purchased from Experimental Animal Center of The Fourth Military Medical University. The mice were fed with common feed and sterile water ad libitum, and housed in $22 \pm 1^{\circ} \mathrm{C}$ with $55 \pm 1 \%$ humidity and a $12 \mathrm{~h}$ light/dark cycles. Athymic nude mice assays were conducted in accordance with the Institutional principles for the concern and use of animals, and the protocol was approved by the ethical committee of The Affiliated Hospital of Southwest Medical University.

SW620 and RKO cells were stably infected with Vector-NC, Vector-G3BP1 and Vector-G3BP1 + sh- $\beta$-catenin (MOI, 10). Then, a total of $5 \times 10^{6}$ cells were resuspended in PBS and injected subcutaneously into the flanks of nude mice $(n=10$ in each group). The animal health and behavior were monitored every 3 days. Mice were euthanized via cervical dislocation and the tumors were removed 28 days after injection. However, the mice were also sacrificed when the tumor diameter was $>1.8 \mathrm{~cm}$. The mice were considered to be dead when the heart and breathing stopped. The largest tumor size was $\sim 2 \mathrm{~cm}^{3}$ and the largest tumor diameter was $\sim 1.2 \mathrm{~cm}$.

Statistical analysis. Data from $\geq 3$ separate experiments are presented as the mean \pm SD. $\mathrm{P}<0.05$ was considered to indicate a statistically significant difference. Data were analyzed using an unpaired or a paired (Fig. 1A and B) two-tailed Student's t-test for two groups, or one way ANOVA followed by Tukey's 
A

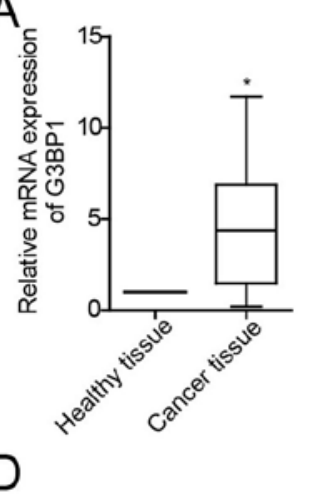

D

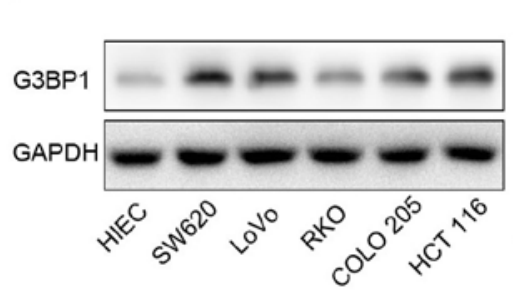

B

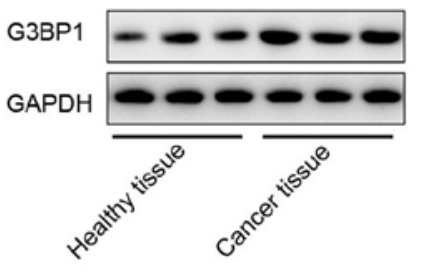

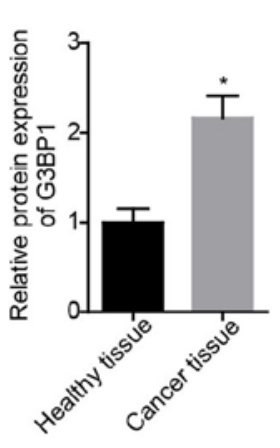

$\mathrm{E}$
C
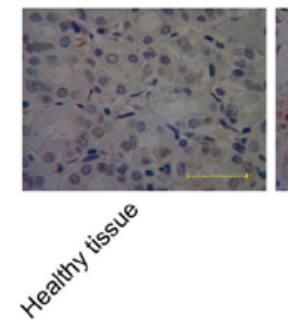

IHC: G3BP1

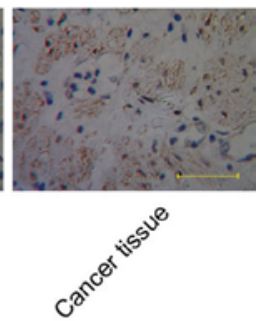

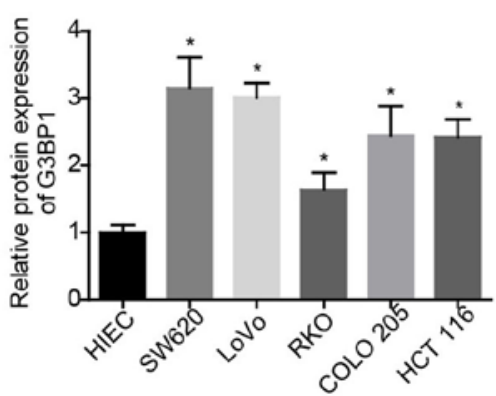

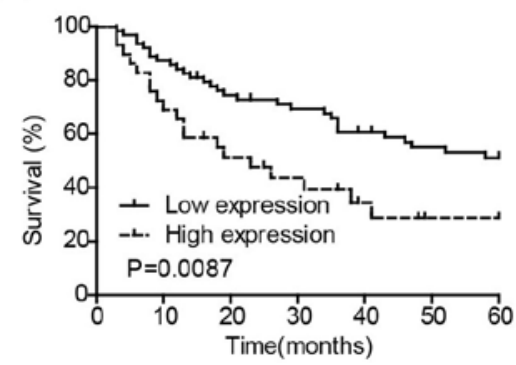

Figure 1. G3BP1 expression was upregulated in colon cancer cells and tissues. (A) Total RNA was extracted from colon cancer tissues and the adjacent healthy colon tissues, and reverse transcription-quantitative PCR was performed to detect G3BP1 mRNA expression (n=20). (B) G3BP1 protein expression in colon cancer tissues and healthy colon tissues was detected using western blotting $(\mathrm{n}=20)$. $\mathrm{P}<0.05$ vs. healthy tissue group. (C) IHC was used to evaluate the different expression patterns of G3BP1 in colon cancer tissues and healthy colon tissues. A representative image of each group is provided ( $\mathrm{n}=20$ ). Scale bar, $100 \mu \mathrm{m}$. (D) G3BP1 protein expression in healthy HIECs and colon cancer cell lines was detected via western blotting. * P<0.05 vs. HIEC group. Data from three independent assays are presented as the mean \pm SD. (E) Kaplan-Meier analysis of the overall survival rates in patients with colon cancer with G3BP1 high or low expression. IHC, immunohistochemistry; G3BP1, Ras-GTPase-activating protein SH3 domain-binding protein 1; HIEC, human intestinal epithelial cell.

post hoc test for multiple groups with SPSS 21.0 software (IBM Corp.). A $\chi^{2}$ test was used to compare the differences in age, sex, vascular invasion, lymph node invasion, differentiation status and TNM stage between patients with G3BP1 high expression or low expression (Table I). The relationship between G3BP1 expression levels and patient overall survival was assessed by using Kaplan-Meier analysis with log rank tests.

\section{Results}

G3BP1 is upregulated in colon cancer tissue specimens and cells. To further examine the mechanism of G3BP1 in the carcinogenesis of colon cancer, its expression profile was assessed in colon cancer tissues and paired para-carcinoma healthy tissues. Increased expression of G3BP1 at both mRNA (Fig. 1A) and protein (Fig. 1B and C) levels was observed in colon cancer tissues compared with healthy tissues. In addition, G3BP1 expression patterns were evaluated in colon cancer cells and healthy colon cells using western blotting. The results demonstrated that G3BP1 protein expression in SW620, RKO, LoVo, COLO 205 and HCT 116 cells was significantly increased compared with HIECs, with RKO demonstrating the lowest, and SW620 having the highest expression levels among these colon cancer cell lines (Fig. 1D). These findings indicated that G3BP1 expression was upregulated in colon cancer tissues and cells.

High expression of G3BPl is closely associated with malignant characterization and poor prognosis in colon cancer. Subsequently, the clinical value of G3BP1 expression in colon cancer was evaluated using Kaplan-Meier curves to determine the overall survival. In addition, a $\chi^{2}$ test was used to analyze the association between G3BP1 expression and the clinicopathologic features in patients with colon cancer. Patients with high G3BP1 expression $(n=29)$ had shorter overall survival time compared with patients with low G3BP1 expression ( $n=63$ ) (Fig. 1E). In addition, G3BP1 expression demonstrated a significant positive association with the incidence rates of vascular invasion and lymph node invasion, as well as differentiation status and TNM stage (Table I). These results suggested that G3BP1 may serve as a marker for the diagnosis and prognosis prediction in patients with colon cancer.

G3BP1 overexpression induces a malignant phenotype of colon cancer cells. As SW620 cells demonstrated the highest expression of G3BP1 and RKO cells presented with the lowest, G3BP1 was knocked down in SW620 cells and overexpressed in RKO cells. Among the three siRNAs targeting G3BP1 gene, si-3 displayed the highest knockdown efficiency in SW620 cells (Fig. 2A), and Vector-G3BP1 significantly enhanced G3BP1 expression in RKO cells (Fig. 2B).

Knockdown of G3BP1 in SW620 cells significantly inhibited the cell proliferative ability (Fig. 2C), while overexpression of G3BP1 in RKO cells significantly enhanced proliferation compared with their corresponding $\mathrm{NCs}$ (Fig. 2D). In addition, knockdown of G3BP1 in SW620 cells induced apoptosis (Fig. 2E), and overexpression of G3BP1 in RKO cells decreased apoptosis (Fig. 2F). Thus, these results indicated that G3BP1 served as an oncogene in colon cancer. 
Table I. Association between clinicopathological characteristics and G3BP1 expression in 92 patients with colorectal cancer.

\begin{tabular}{|c|c|c|c|c|}
\hline \multirow[b]{2}{*}{ Characteristics } & \multirow[b]{2}{*}{ Total no. patients } & \multicolumn{2}{|c|}{ G3BP1 expression } & \multirow[b]{2}{*}{ P-value } \\
\hline & & Low $(n=63)$ & High $(n=29)$ & \\
\hline Age, years & & & & 0.541 \\
\hline$<60$ & 55 & 39 & 16 & \\
\hline$\geq 60$ & 37 & 24 & 13 & \\
\hline Sex & & & & 0.732 \\
\hline Male & 50 & 35 & 15 & \\
\hline Female & 42 & 28 & 14 & \\
\hline Vascular invasion & & & & 0.032 \\
\hline No & 70 & 52 & 18 & \\
\hline Yes & 22 & 11 & 11 & \\
\hline Lymph node invasion & & & & 0.013 \\
\hline Absent & 61 & 47 & 14 & \\
\hline Present & 31 & 16 & 15 & \\
\hline Differentiation status & & & & 0.033 \\
\hline High & 66 & 42 & 24 & \\
\hline Low & 26 & 21 & 5 & \\
\hline TNM stage & & & & 0.044 \\
\hline I-II & 72 & 53 & 19 & \\
\hline III-IV & 20 & 10 & 10 & \\
\hline
\end{tabular}

G3BP1, Ras-GTPase-activating protein SH3 domain-binding protein 1.

A
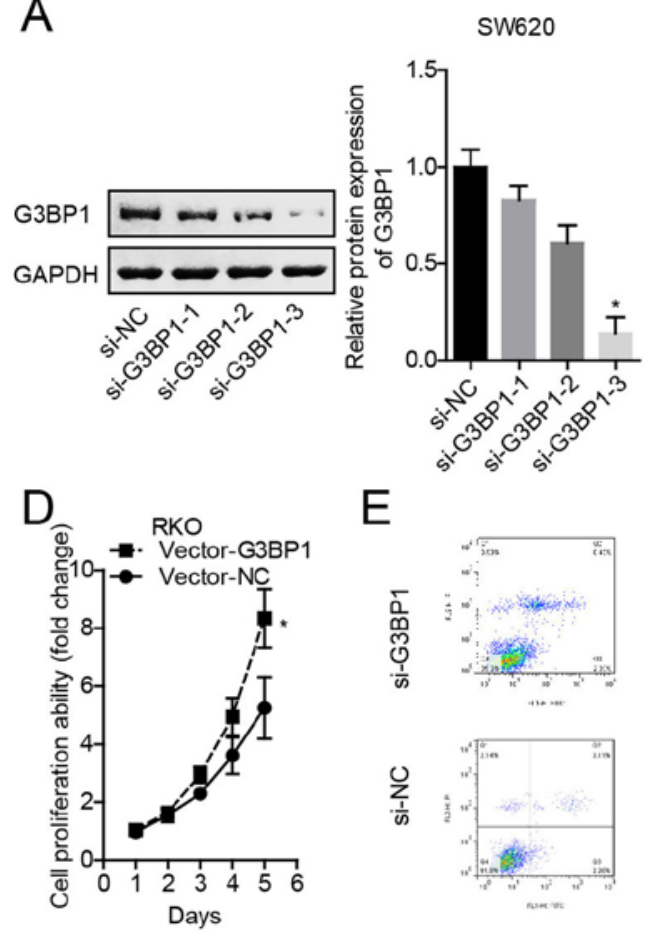

B

E

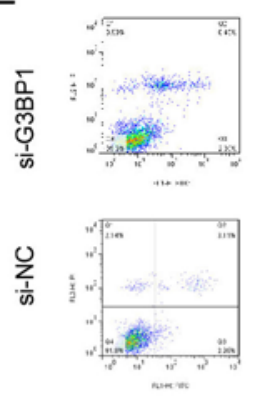

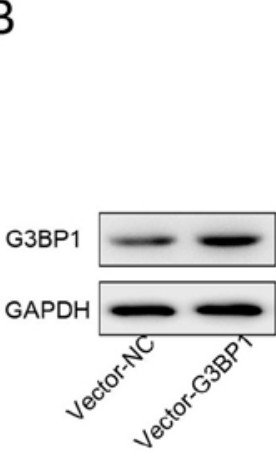

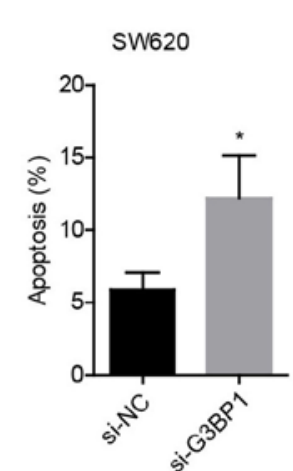

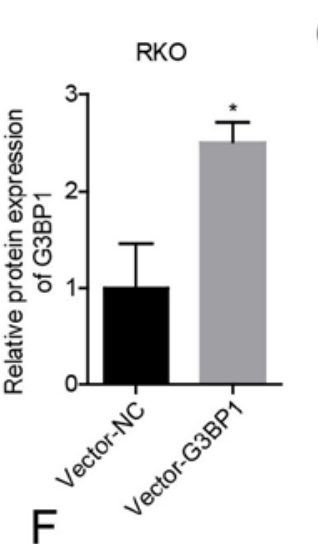

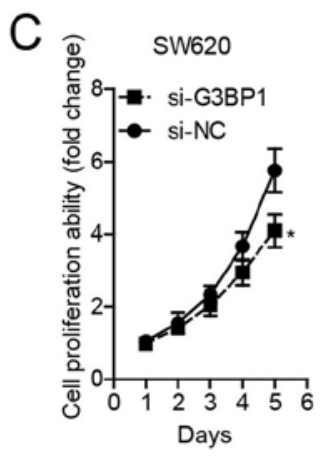

$\mathrm{F}$
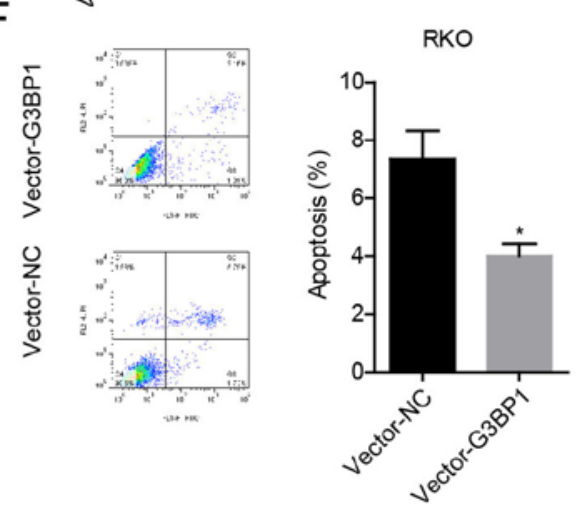

Figure 2. G3BP1 overexpression promotes cell proliferation and inhibits cell apoptosis in colon cancer. SW620 cells were transfected with si-G3BP1 or si-NC, while RKO cells were infected with Vector-G3BP1 or Vector-NC. G3BP1 protein expression was determined using western blotting in (A) transfected SW620 cells and (B) infected RKO cells. Effects of altered G3BP1 expression on (C) SW620 and (D) RKO cell proliferation were assessed with a Cell Counting Kit-8 assay. Effects of altered G3BP1 expression on cell apoptosis was assessed using FACS assay in (E) transfected SW620 cells and (F) infected RKO cells. Data from three independent assays are presented as the mean \pm SD. ${ }^{*} \mathrm{P}<0.05$ vs. si-NC or Vector-NC groups. G3BP1, Ras-GTPase-activating protein SH3 domain-binding protein 1; siRNA, small interfering RNA; NC, negative control. 

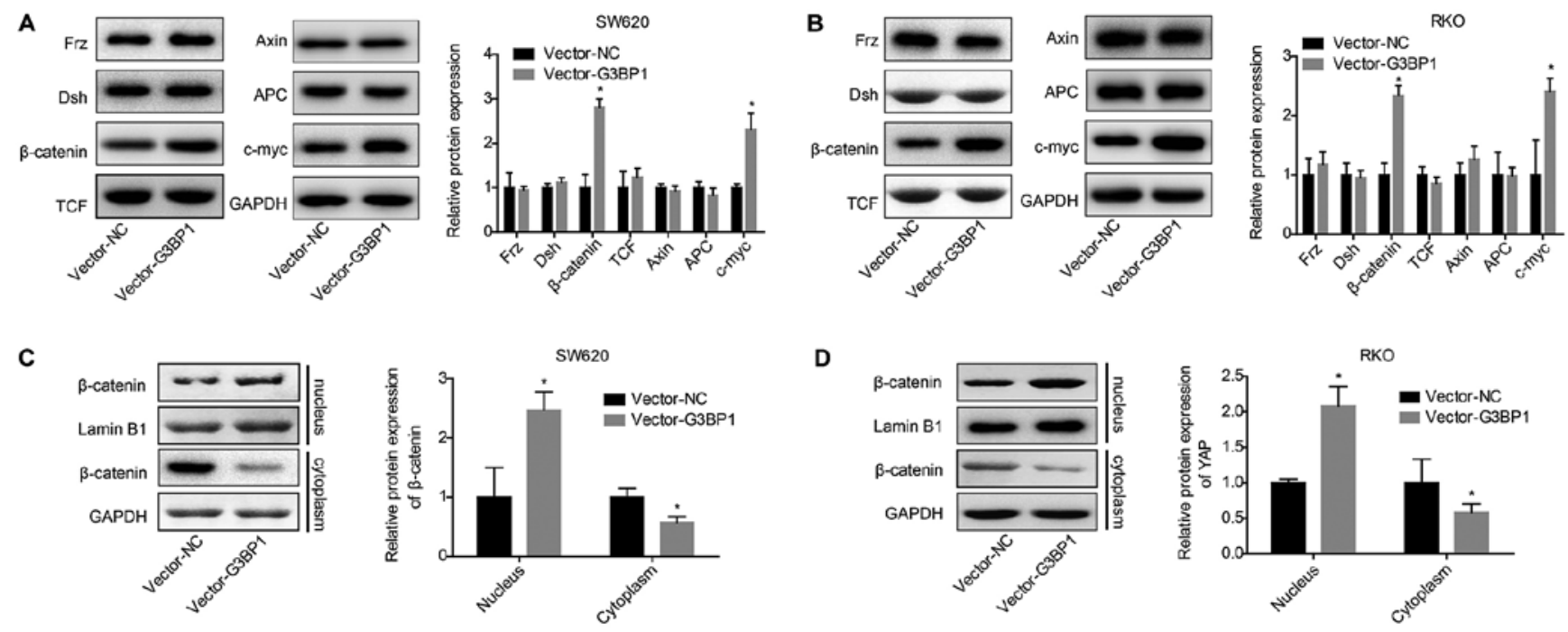

E SW620

$\mathbf{F}$

RKO
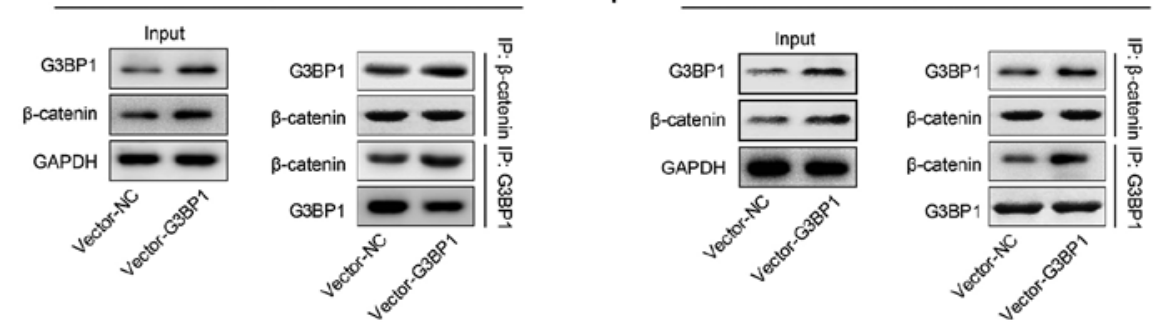

Figure 3. G3BP1 activates $\beta$-catenin in colon cancer cells. SW620 and RKO cells were transfected with Vector-NC or Vector-G3BP1 and the following assays were performed. Protein expression levels of Frz, Dsh, TCF, APC, Axin, c-myc and $\beta$-catenin were detected via western blotting after $48 \mathrm{~h}$ of transfection in (A) SW620 and (B) RKO cells. Cell nuclear and cytoplasm proteins were extracted for western blot analysis to detect $\beta$-catenin levels in nuclear (Lamin B1 was used as an internal reference for nuclear protein) and cytoplasmic fractions (GAPDH was used as an internal reference for cytoplasm protein) in (C) SW620 and (D) RKO cells. Interaction between G3BP1 and $\beta$-catenin was determined using a co-IP assay in (E) SW620 and (F) RKO cells. Data from three independent assays are presented as the mean \pm SD. ${ }^{*} \mathrm{P}<0.05$ vs. Vector-NC group. co-IP, co-immunoprecipitation; G3BP1, Ras-GTPase-activating protein SH3 domain-binding protein 1; NC, negative control; Frz, Frizzled; Dsh, Dishevelled; TCF, transcription factor; Axin, axis inhibitor; APC, adenomatous polyposis coil.

Overexpression of G3BP1 translocates $\beta$-catenin into nucleus of colon cancer cells. To investigate whether Wnt/ $\beta$-catenin signaling is involved in G3BP1-mediated colon cancer progression, a series of assays were conducted to assess the association between $\mathrm{Wnt} / \beta$-catenin signaling and G3BP1. The western blotting results identified that G3BP1 overexpression could significantly increase the expression levels of $\beta$-catenin and c-myc, whereas it had no notable effect on the expression levels of Frz, Dsh, APC, Axin and TCF in SW620 (Fig. 3A) and RKO cells (Fig. 3B). Moreover, G3BP1 overexpression significantly facilitated the translocation of $\beta$-catenin protein from the cytoplasm to the nucleus (Fig. 3C and D). The co-IP assay results also demonstrated that G3BP1 could directly or indirectly bind to $\beta$-catenin, and the overexpression of G3BP1 markedly enhanced their interaction (Fig. 3E and F). Therefore, it was suggested that G3BP1 overexpression could induce the activation of $\beta$-catenin signaling.

G3BP1 facilitates the progression of colon cancer by upregulating $\beta$-catenin. Finally, the role of $\beta$-catenin in G3BP1-mediated cell proliferation enhancement and apoptosis suppression in colon cancer was assessed. The expression of $\beta$-catenin was significantly decreased following cell transfection with si- $\beta$-catenin in both SW620 and RKO cells compared with the si-NC group (Fig. 4A and B). It was found that overexpression of G3BP1 significantly enhanced $\beta$-catenin expression, but this tendency was significantly rescued by sh- $\beta$-catenin infection in SW620 and RKO cells (Fig. 4C and D). Moreover, knockdown of $\beta$-catenin weakened the role of G3BP1 in cell proliferation enhancement (Fig. 4E and F) and cell apoptosis inhibition (Fig. 4G and H). The results also demonstrated that G3BP1 overexpression significantly enhanced the tumorigenesis of SW620 and RKO cells, whereas this effect was abolished when $\beta$-catenin was stably knocked down (Fig. 5A and B). Collectively, the results indicated that G3BP1 facilitated the progression of colon cancer in a $\beta$-catenin-dependent manner.

\section{Discussion}

Identification and investigation of dysregulated genes are important to understand the pathogenesis of cancer types and for evaluating novel therapeutic targets. Although G3BP1 has been reported to be upregulated in colon cancer, and it has been shown that knockdown of G3BP1 significantly inhibits cell proliferation and improves the chemosensitivity of HCT116 cells (18), the clinical significance and mechanisms of G3BP1 in colon cancer development remain largely unknown.

In the present study, it was identified that G3BP1 was significantly upregulated in colon cancer tissues and cells, and 

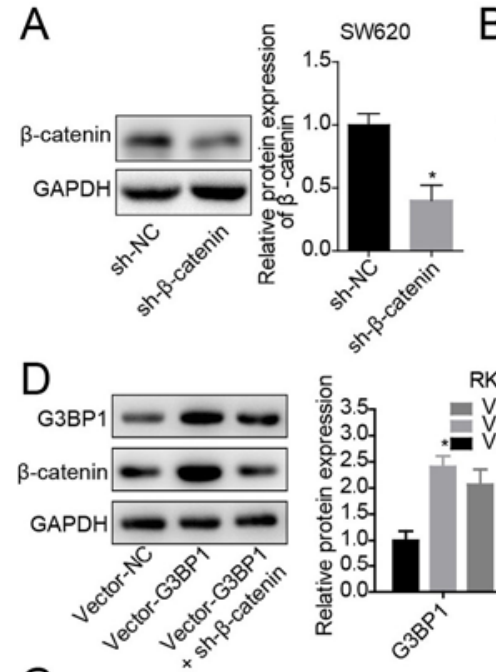

G

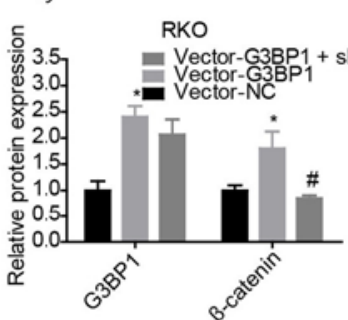

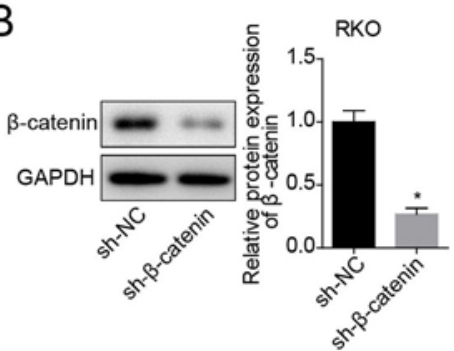

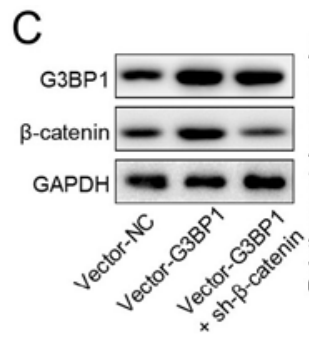

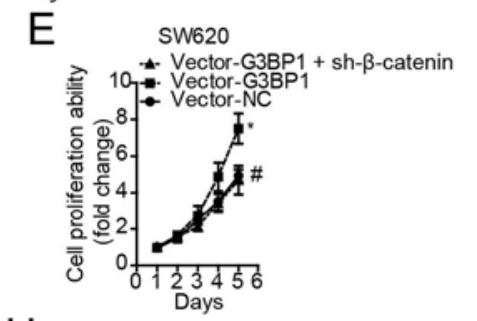

$\mathrm{H}$

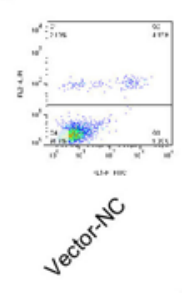

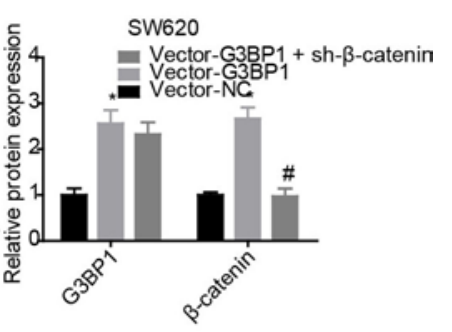

F RKO

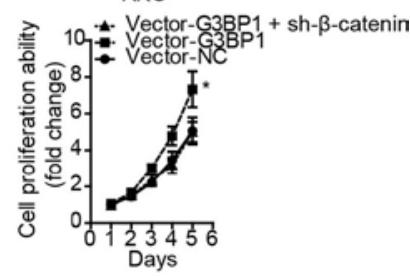

RKO
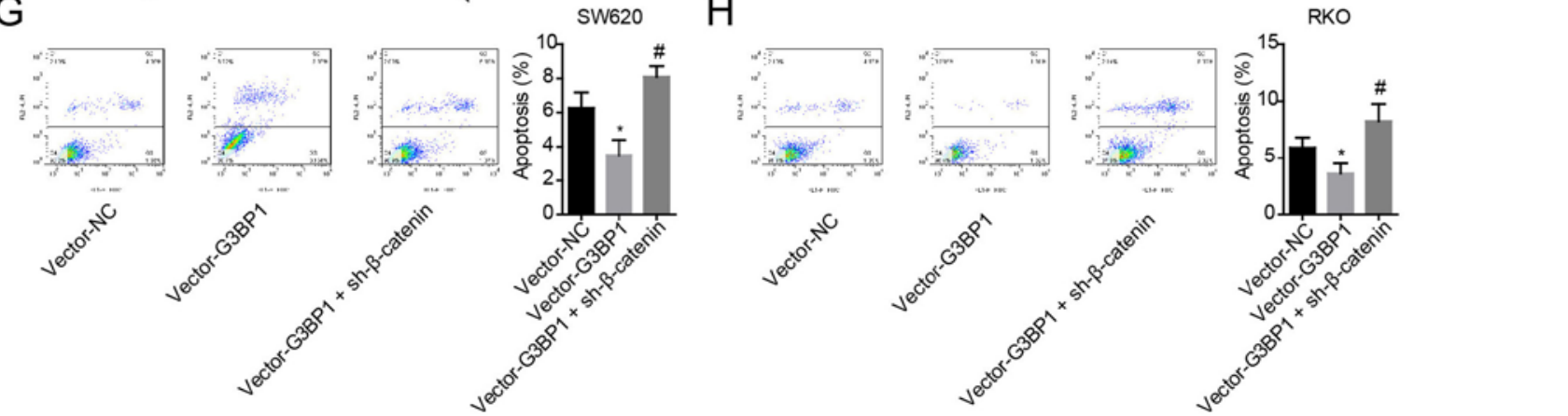

Figure 4. Knockdown of $\beta$-catenin abolishes the effect of G3BP1 on cell proliferation and apoptosis. SW620 and RKO cells were transfected with Vector-NC, Vector-G3BP1 and Vector-G3BP1 + sh- $\beta$-catenin, then the following assays were performed. Protein expression of $\beta$-catenin was detected using western blotting after $48 \mathrm{~h}$ of transfection in (A) SW620 and (B) RKO cells. Cells were harvested for western blotting to measure $\beta$-catenin protein expression after $48 \mathrm{~h}$ of transfection in (C) SW620 and (D) RKO cells. Cell Counting Kit-8 assays were performed for five consecutive days to detect proliferation in (E) SW620 and (F) RKO cells. (G) SW620 and (H) RKO cells were collected for FACS to detect cell apoptosis after $48 \mathrm{~h}$ cell transfection. Data from three independent assays are presented as the mean $\pm \mathrm{SD}$. " $\mathrm{P}<0.05$ vs. Vector-NC group; ${ }^{\#} \mathrm{P}<0.05$ vs. Vector-G3BP1 group. G3BP1, Ras-GTPase-activating protein SH3 domain-binding protein 1; NC, negative control; siRNA, small interfering RNA.

A

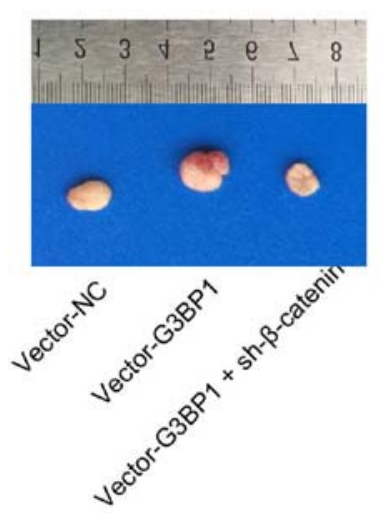

SW620

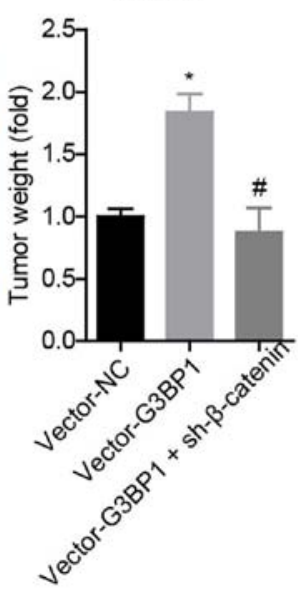

B

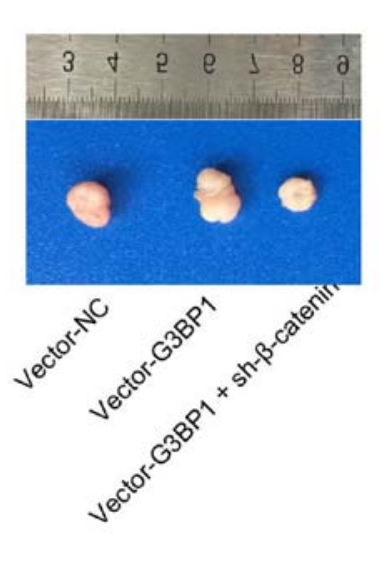

RKO

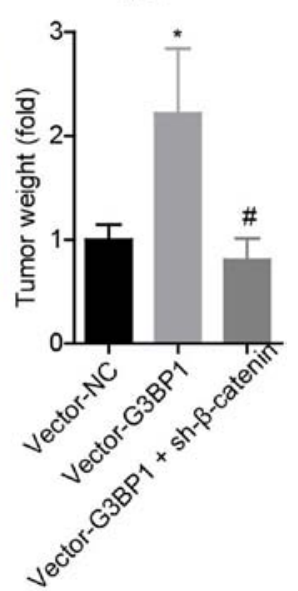

Figure 5. Knockdown of $\beta$-catenin abolishes the effect of G3BP1 on cell tumorigenesis improvement. (A) SW620 and (B) RKO cells were stably transfected with Vector-NC, Vector-G3BP1 and Vector-G3BP1 + sh- $\beta$-catenin, and these were injected into nude mice. Tumor average weights from each group are presented as the mean $\pm \mathrm{SD}(\mathrm{n}=5)$ * $\mathrm{P}<0.05$ vs. Vector-NC group; ${ }^{\text {P }}<0.05$ vs. Vector-G3BP1 group. G3BP1, Ras-GTPase-activating protein SH3 domain-binding protein 1 ; NC, negative control; shRNA, short hairpin RNA.

that high expression of G3BP1 was closely associated with vascular invasion, lymph node invasion, the differentiation status and TNM stage of colon cancer, as well as predicted a poor prognosis. These findings indicated a crucial value of G3BP1 in predicting the clinic process and outcome of patients with colon cancer. In line with the current results, 
Dou et al (27) revealed that G3BP1 was upregulated in hepatocellular carcinoma (HCC), and its high expression was significantly associated with the poor prognosis of patients with HCC. Furthermore, Min et al (15) observed that G3BP1 was upregulated in gastric cancer at the protein level, and that the G3BP1 expression pattern was positively correlated with tumor size, TNM stage, vascular invasion and lymph node metastasis, but negatively associated with the overall survival of patients. However, it has also been reported that G3BP1 expression is decreased in gastric cancer, and is significantly associated with age and disease stage, but demonstrates no significant correlation with cancer metastasis in gastric cancer (28). Therefore, different sources of tissue samples may influence the varying roles of G3BP1 in carcinogenesis.

G3BP1 is an important marker of stress granules (SG) and is induced by several stress factors, such as chemotherapy, heat shock and hypoxia (29). The formation of SG can protect cells from apoptosis by inhibiting the stress-responsive MAPK pathways (30). Moreover, G3BP1 serves an important role in the regulation of a variety of growth-related signaling pathways, including NF- $\mathrm{B}$, Ras and PI3K signaling pathways and the ubiquitin proteasome system (31-34). G3BP1 also negatively modulates p53 expression (35), a tumor suppressive gene found in multiple tumor types, including colon cancer (36). Thus, G3BP1 may be implicated in carcinogenesis. Previous studies have reported that G3BP1 significantly promotes the progression of several types of cancer. For instance, Winslow et al (10) revealed that G3BP1 was an important factor for breast cancer cell proliferation, and knockdown of G3BP1 significantly inhibited cell proliferation by upregulating PMP22. In addition, knockdown of G3BP1 prevents SG formation and tumor invasion, as well as lung metastasis in mouse sarcoma xenografts models (37). It has also been shown that G3BP1 knockdown significantly inhibits the metastasis capacity of HCC cells in vitro and in vivo (27). In the present study, it was identified that G3BP1 overexpression could significantly enhanced the proliferation and tumorigenesis of colon cancer cells and decreased cell apoptosis, indicating that G3BP1 functioned as an oncogene in colon cancer progression.

While it has been reported that the Wnt/ $\beta$-catenin signaling pathway serves an important role in the progression of colon cancer $(19,20)$, the mechanism underlying its hyperactivation is not fully understood. In the current study, it was demonstrated that the increased expression levels of $\beta$-catenin and c-myc proteins, together with the nuclear accumulation of $\beta$-catenin protein, could be induced by G3BP1 overexpression, with no notable change in the expression levels of Frz, Dsh, TCF, Axin and APC. In addition, it was identified that the G3BP1 protein combined with $\beta$-catenin protein. These results suggest that G3BP1 can activate $\beta$-catenin signaling. However, by contrast, G3BP1 has been revealed to exert an mRNA-degrading role in regulating $\beta$-catenin in response to $\mathrm{Wnt} 3 \mathrm{a}$ in totipotent mouse embryonic F9 cells (23), which may be caused by the different cell contents. Guitard et al (17) reported that the expression patterns of G3BPs and c-myc proteins (c-mycl and c-myc2) were similar in human head and neck tumors, which further indicates that G3BPs are involved in c-myc mRNA stabilization, thereby facilitating cell proliferation (32). Similarly, the present results suggested that G3BP1 positively regulated the expression of c-myc protein, and this may be associated with the stability of c-myc mRNA. It was identified that the enhancements in cell proliferation and tumorigenesis, as well as the decreases in cell apoptosis induced by G3BP1 overexpression were significantly rescued by $\beta$-catenin knockdown, suggesting that G3BP1 facilitated colon cancer via activating $\beta$-catenin signaling.

In conclusion, the present study demonstrated that G3BP1 was upregulated in colon cancer, and the high expression of G3BP1 predicted malignant clinical process and poor outcome in patients with colon cancer. Mechanically, overexpression of G3BP1 promoted the progression of colon cancer by activating $\beta$-catenin signaling. Collectively, the present study provides a novel understanding of the roles of G3BP1 in colon cancer progression.

\section{Acknowledgements}

Not applicable.

\section{Funding}

The current study was supported by a research grant for Doctors of the Affiliated Hospital of Southwest Medical University and Luzhou People's Government-Southwest Medical University Cooperative Scientific Research Project (grant no. 2019LZXNDJ26).

\section{Availability of data and materials}

All data generated or analyzed during this study are included in this published article.

\section{Authors' contributions}

YL and JW conceived the idea of the study and performed the experiments, as well as wrote the manuscript. SZ and JL performed the data analyses. WD performed parts of the data analyses. YL revised the manuscript. All authors read and approved the final manuscript.

\section{Ethics approval and consent to participate}

Experiments involving human samples were performed in accordance with the Helsinki Declaration and were approved by the ethical committee of The Affiliated Hospital of Southwest Medical University. All patients signed the informed consent.Athymic nude mice assays were performed in accordance with the Institutional principles for the concern and use of animals, and the protocol was approved by the ethical committee of The Affiliated Hospital of Southwest Medical University.

\section{Patient consent for publication}

Not applicable.

\section{Competing interests}

The authors declare that they have no competing interests. 


\section{References}

1. Siegel RL, Miller KD and Jemal A: Cancer statistics, 2019. CA CAncer J Clin 69: 7-34, 2019.

2. Miller KD, Siegel RL, Lin CC, Mariotto AB, Kramer JL, Rowland JH, Stein KD, Alteri R and Jemal A: Cancer treatment and survivorship statistics, 2016. CA Cancer J Clin 66: 271-289, 2016.

3. Brenner H, Kloor M and Pox CP: Colorectal cancer. Lancet 383: 1490-1502, 2014

4. Pothuraju R, Rachagani S, Krishn SR, Chaudhary S, Nimmakayala RK, Siddiqui JA, Ganguly K, Lakshmanan I, Cox JL, Mallya K, etal: Molecularimplications of MUC5AC-CD44 axis in colorectal cancer progression and chemoresistance. Mol Cancer 19: 37, 2020.

5. Briggs SD, Bryant SS, Jove R, Sanderson SD and Smithgall TE: The Ras GTPase-activating protein (GAP) is an SH3 domain-binding protein and substrate for the Src-related tyrosine kinase, Hck. J Biol Chem 270: 14718-14724, 1995.

6. Atlas R, Behar L, Elliott E and Ginzburg I: The insulin-like growth factor mRNA binding-protein IMP-1 and the Ras-regulatory protein $\mathrm{G} 3 \mathrm{BP}$ associate with tau mRNA and $\mathrm{HuD}$ protein in differentiated P19 neuronal cells. J Neurochem 89: 613-626, 2004

7. Taniuchi K, Nishimori I and Hollingsworth MA: The N-terminal domain of G3BP enhances cell motility and invasion by posttranscriptional regulation of BART. Mol Cancer Res 9: 856-866, 2011.

8. French J, Stirling R, Walsh M and Kennedy HD: The expression of Ras-GTPase activating protein SH3 domain-binding proteins, G3BPs, in human breast cancers. Histochem J 34: 223-231, 2002.

9. Zheng H, Zhan Y, Zhang Y, Liu S, Lu J, Yang Y, Wen Q and Fan S: Elevated expression of G3BP1 associates with YB1 and p-AKT and predicts poor prognosis in nonsmall cell lung cancer patients after surgical resection. Cancer Med 8: 6894-6903, 2019.

10. Winslow S, Leandersson K and Larsson C: Regulation of PMP22 mRNA by G3BP1 affects cell proliferation in breast cancer cells. Mol Cancer 12: 156, 2013.

11. Irvine K, Stirling R, Hume D and Kennedy D: Rasputin, more promiscuous than ever: A review of G3BP. Int J Dev Biol 48: 1065-1077, 2004.

12. Kociok N, Esser P, Unfried K, Parker F, Schraermeyer U, Grisanti S, Toqué B and Heimann K: Upregulation of the RAS-GTPase activating protein (GAP)-binding protein (G3BP) in proliferating RPE cells. J Cell Biochem 74: 194-201, 1999.

13. Oi N, Yuan J, Malakhova M, Luo K, Li Y, Ryu J, Zhang L, Bode AM, Xu Z, Li Y, et al: Resveratrol induces apoptosis by directly targeting Ras-GTPase-activating protein SH3 domain-binding protein 1. Oncogene 34: 2660-2671, 2015.

14. Zhang H, Ma Y, Zhang S, Liu H, He H, Li N, Gong Y, Zhao S, Jiang JD and Shao RG: Involvement of Ras GTPase-activating protein SH3 domain-binding protein 1 in the epithelialto-mesenchymal transition-induced metastasis of breast cancer cells via the Smad signaling pathway. Oncotarget 6: 17039-17053, 2015.

15. Min L, Ruan Y, Shen Z, Jia D, Wang X, Zhao J, Sun Y and $\mathrm{Gu} \mathrm{J}$ : Overexpression of Ras-GTPase-activating protein $\mathrm{SH} 3$ domain-binding protein 1 correlates with poor prognosis in gastric cancer patients. Histopathology 67: 677-688, 2015.

16. Shim JH, Su ZY, Chae JI, Kim DJ, Zhu F, Ma WY, Bode AM, Yang CS and Dong Z: Epigallocatechin gallate suppresses lung cancer cell growth through Ras-GTPase-activating protein SH3 domain-binding protein 1. Cancer Prev Res (Phila) 3: 670-679, 2010.

17. Guitard E, Parker F, Millon R, Abecassis J and Tocque B: G3BP is overexpressed in human tumors and promotes $\mathrm{S}$ phase entry. Cancer Lett 162: 213-221, 2001.

18. Zhang H, Zhang S, He H, Zhao W, Chen J and Shao RG: GAP161 targets and downregulates G3BP to suppress cell growth and potentiate cisplaitin-mediated cytotoxicity to colon carcinoma HCT116 cells. Cancer Sci 103: 1848-1856, 2012

19. Zhang J,Li Q,Xue B and He R: MALAT1 inhibits the Wnt/ $\beta$-catenin signaling pathway in colon cancer cells and affects cell proliferation and apoptosis. Bosn J Basic Med Sci 20: 357-364, 2019.
20. Shiizaki K, Kido K and Mizuta Y: Insight into the relationship between aryl-hydrocarbon receptor and $\beta$-catenin in human colon cancer cells. PLoS One 14: e0224613, 2019.

21. Kim JY, Park G, Krishnan M, Ha E and Chun KS: Selective Wnt $/ \beta$-catenin Small-molecule Inhibitor CWP232228 Impairs Tumor Growth of Colon Cancer. Anticancer Res 39: 3661-3667, 2019.

22. Sebio A, Kahn M and Lenz HJ: The potential of targeting Wnt/ $\beta$-catenin in colon cancer. Expert Opin Ther Targets 18: 611-615, 2014.

23. Bikkavilli RK and Malbon CC: Arginine methylation of G3BP1 in response to Wnt3a regulates $\beta$-catenin mRNA. J Cell Sci 124: 2310-2320, 2011

24. Xin B, He X, Wang J, Cai J, Wei W, Zhang T and Shen X: Nerve growth factor regulates CD133 function to promote tumor cell migration and invasion via activating ERK1/2 signaling in pancreatic cancer. Pancreatology 16: 1005-1014, 2016.

25. Livak KJ and Schmittgen TD: Analysis of relative gene expression data using real-time quantitative PCR and the 2(-Delta Delta C(T)) method. Methods 25: 402-408, 2001.

26. Duquet A, Melotti A, Mishra S, Malerba M, Seth C, Conod A and Ruiz I Altaba A: A novel genome-wide in vivo screen for metastatic suppressors in human colon cancer identifies the positive WNT-TCF pathway modulators TMED3 and SOX12. EMBO Mol Med 6: 882-901, 2014.

27. Dou N, Chen J, Yu S, Gao Y and Li Y: G3BP1 contributes to tumor metastasis via upregulation of Slug expression in hepatocellular carcinoma. Am J Cancer Res 6: 2641-2650, 2016.

28. Beheshtizadeh M and Moslemi E: Analysis of G3BP1 and VEZT expression in gastric cancer and their possible correlation with tumor clinicopathological Factors. J Gastric Cancer 17: 43-51, 2017.

29. Tourriere H, Chebli K, Zekri L, Courselaud B, Blanchard JM, Bertrand $\mathrm{E}$ and Tazi J: The RasGAP-associated endoribonuclease G3BP assembles stress granules. J Cell Biol 160: 823-831, 2003.

30. Arimoto K, Fukuda H, Imajoh-Ohmi S, Saito H and Takekawa M: Formation of stress granules inhibits apoptosis by suppressing stress-responsive MAPK pathways. Nat Cell Biol 10: 1324-1332, 2008.

31. Barnes CJ, Li F, Mandal M, Yang Z, Sahin AA and Kumar R: Heregulin induces expression, ATPase activity, and nuclear localization of G3BP, a Ras signaling component, in human breast tumors. Cancer Res 62: 1251-1255, 2002.

32. Gallouzi IE, Parker F, Chebli K, Maurier F, Labourier E, Barlat I, Capony JP, Tocque B and Tazi J: A novel phosphorylation-dependent RNase activity of GAP-SH3 binding protein: A potential link between signal transduction and RNA stability. Mol Cell Biol 18: 3956-3965, 1998.

33. Soncini C, Berdo I and Draetta G: Ras-GAP SH3 domain binding protein (G3BP) is a modulator of USP10, a novel human ubiquitin specific protease. Oncogene 20: 3869-3879, 2001.

34. Huang Y, Wernyj RP, Norton DD, Precht P, Seminario MC and Wange RL: Modulation of specific protein expression levels by PTEN: Identification of AKAP121, DHFR, G3BP, Rap1, and RCC1 as potential targets of PTEN. Oncogene 24: 3819-3829, 2005.

35. Kim MM, Wiederschain D, Kennedy D, Hansen E and Yuan ZM: Modulation of p53 and MDM2 activity by novel interaction with Ras-GAP binding proteins (G3BP). Oncogene 26: 4209-4215, 2007.

36. Chen G, Zhou T, Li Y, Yu Z and Sun L: p53 target miR-29c-3p suppresses colon cancer cell invasion and migration through inhibition of PHLDB2. Biochem Biophys Res Commun 487: 90-95, 2017.

37. Somasekharan SP, El-Naggar A, Leprivier G, Cheng H, Hajee S, Grunewald TG, Zhang F, Ng T, Delattre O, Evdokimova V, et al: YB-1 regulates stress granule formation and tumor progression by translationally activating G3BP1. J Cell Biol 208: 913-929, 2015.

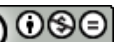

This work is licensed under a Creative Commons Attribution-NonCommercial-NoDerivatives 4.0 International (CC BY-NC-ND 4.0) License. 